\title{
Jugendliche und junge Erwachsene in der ambulanten Suchthilfe. Empirische Befunde vor dem Hintergrund des Kinder- und Jugendhilfegesetzes
}

\author{
J. Kalke \\ C. Schütze \\ M. Kloss \\ M.-S. Martens \\ P. Raschke
}

Adolescents and Young Adults in Outpatient Addiction Services. Empirical

Findings against the Background of the Act of Help for Children and Young People

\section{Zusammenfassung}

Dieser Beitrag beschäftigt sich mit der Frage, welche Bedeutung Jugendliche und junge Erwachsene für die ambulante Suchthilfe besitzen. Diese Fragestellung hat vor dem Hintergrund des Kinder- und Jugendhilfegesetzes (KJHG) eine versorgungspolitische Relevanz, denn es wird immer wieder diskutiert, ob die ambulante Suchthilfe auch Aufgabenbereiche der erzieherischen Hilfen und Jugendhilfen wahrnimmt und ob es spezielle Angebote für drogengebrauchende Jugendliche geben sollte, die nach dem KJHG finanziert werden. Als empirische Grundlage für die Analyse wurden Daten aus Schleswig-Holstein ausgewertet. Dort findet seit einigen Jahren ein systematisches Monitoring der Klienten und Tätigkeiten der ambulanten Suchthilfe statt. Die statistische Analyse zeigt, dass es sich bei $5 \%$ der Klienten um Jugendliche (14 bis 17 Jahre) und bei 19\% um junge Erwachsene (18 bis 26 Jahre) handelt. Die „Konsumprofile“ dieser beiden Gruppen weichen stark voneinander ab; bei der erstgenannten Gruppe dominieren die Cannabisgebraucher, in der zweiten gibt es viele Heroinabhängige. Entsprechend stellt sich die soziale Situation dar: Die meisten Jugendlichen sozial integriert; bei den 18- bis 26-Jährigen befindet sich dagegen ein erheblicher Teil in einer schlechten sozialen Lage. Ebenfalls ist das Inanspruchnahmeverhalten beider Gruppen verschieden: Die Jugendlichen sind deutlich kürzer, mit weniger Kontakten und geringerem Zeitaufwand in einer Betreuung als die jungen Erwachsenen. Vor diesem empirischen Hintergrund wird eine (theoretische) Zuordnung der Klientel und der empfangenen Hilfen zum Leistungskatalog des Kinderund Jugendhilfegesetzes (KJHG) vorgenommen. Damit soll eine Grundlage für weitere Diskussionen um die Aufgabenvertei-

\section{Abstract}

The present article focuses on adolescents and young adults and their importance in the context of outpatient addiction support services. Against the background of the Act of Help for Children and Young People (Kinder- und Jugendhilfegesetz - KJHG), this is a highly relevant issue in the field of health care policy. There is an ongoing discussion whether outpatient addiction support should include responsibilities of educational and youth services and whether there should be special programmes for drug using adolescents funded according to the KJHG. The analysis is based on empirical data from Schleswig-Holstein, where systematic monitoring of clients and activities of outpatient addiction services has been going on since a few years. The statistical analysis shows that $5 \%$ of the clients are adolescents (14-17 years) and $19 \%$ young adults ( $18-26$ years). Both groups strongly differ in their consumption profiles; cannabis users are predominant in the first group and there are many heroin users in the second group. The social situation corresponds to this finding: Most adolescents are socially integrated; in the age group $18-26$ years, a considerable number lives in adverse social conditions. The utilisation of support facilities is also different in both groups: Adolescents utilise care facilities for considerably shorter periods, with fewer contacts and in a less time consuming way than young adults. Against this empirical background, clients and the support they received are (theoretically) assigned to the service catalogue of the Act of Help for Children and Young People (KJHG) with the aim to build a basis for further discussions concerning the task distribution of addiction support services and help for young people as well as the development of specific help offers. 
lung von Sucht- und Jugendhilfe sowie die Weiterentwicklung spezifischer Angebote geschaffen werden.

\section{Schliuisselwörter}

Ambulante Suchthilfe $\cdot$ Jugendliche $\cdot$ junge Erwachsene $\cdot$ Kinderund Jugendhilfegesetz
Key words

Outpatient addiction support · adolescents $\cdot$ young adults $\cdot$ Act of Help for Children and Young People

\section{Einführung und Fragestellung}

Dieser Beitrag beschäftigt sich mit der Frage, welche Bedeutung Jugendliche und junge Erwachsene für die ambulante Suchthilfe besitzen. Wie hoch ist ihr Anteil an der Gesamtzahl der Klienten? Wie sehen ihr biografischer Hintergrund und ihre aktuelle soziale Situation aus? Woher kommen sie, wie lange bleiben sie, welche Leistungen erhalten sie und wohin werden sie vermittelt? Diese Fragen nach dem Hilfebedarf haben vor dem Hintergrund des Kinder- und Jugendhilfegesetzes (KJHG), in dem die Aufgaben der Jugendhilfe definiert sind, eine versorgungspolitische Relevanz. Denn es wird immer wieder die Frage gestellt, ob die ambulante Suchthilfe auch Aufgabenbereiche der erzieherischen Hilfen und Jugendhilfen wahrnimmt und ob es spezielle Angebote für drogengebrauchende Jugendliche geben sollte, die nach dem KJHG finanziert werden $[1,2]$. Da es sich darüber hinaus beim KJHG um ein präventiv orientiertes Leistungsgesetz mit einer pädagogischen Ausrichtung handelt, stellt sich insgesamt die Frage, ob die Suchthilfe zumindest teilweise präventive Funktionen (Frühinterventionen) für junge Menschen wahrnimmt. In diesem Zusammenhang gibt es eine - schon länger andauernde - fachliche Diskussion über die Zusammenarbeit, die Schnittmenge sowie die originären Aufgabenbereiche von Sucht- und Jugendhilfe [1, 2]. Dabei geht es auch um Finanzierungsfragen, denn für Leistungen nach dem KJHG sind primär die Kommunen zuständig.

Grundsätzlich formuliert die Jugendhilfe den Anspruch, das Regelversorgungssystem für Heranwachsende und junge Menschen darzustellen. Das Suchthilfesystem mit seinen Angeboten für jüngere Klienten konnte sich seit Anfang der 70er-Jahre parallel dazu entwickeln, weil die Jugendhilfe nicht in der Lage war, den spezifischen Anforderungen des zunehmenden Drogenkonsums unter Jugendlichen adäquat zu begegnen [3]. Seitdem haben sich ganz unterschiedliche Formen der Zusammenarbeit zwischen Jugend- und Suchthilfe entwickelt. So gibt es in einigen Landkreisen - als ein Beispiel für eine sehr enge Kooperation Versorgungsverträge zwischen dem Kreisjugendamt und den Suchtberatungsstellen, die bestimmte Leistungen des KJHG für besondere Zielgruppen übernehmen [3]. Auch gibt es Modelle, bei denen im Rahmen eines vom Jugendamt eingeleiteten Hilfeprozesses gemäß §36 KJHG (Mitwirkung, Hilfeplan) die Drogenhilfe systematisch einbezogen wird. Andernorts beschränkt sich die Zusammenarbeit dagegen auf einen unverbindlichen Erfahrungsaustausch.

Die Regelungen des KJHG beziehen sich auf Personen bis zu einem Alter von einschließlich 26 Jahren. Dabei werden nach den Begriffsbestimmungen des KJHG die folgenden Altersgruppen unterschieden $(\S 7)$ :
- Kind: wer noch nicht 14 Jahre alt ist,

- Jugendlicher: wer 14, aber noch nicht 18 Jahre alt ist und

- junger Erwachsener (Volljähriger): wer 18, aber noch nicht 27 Jahre alt ist.

Das KJHG beschreibt von seinem Wortlaut her keine Leistungen für substanzgebrauchende Kinder und Jugendliche, es schließt aber solche auch nicht explizit aus. Das KJHG bietet in dieser Hinsicht interpretatorischen Spielraum. Folgende Leistungen kommen für Kinder und Jugendliche mit Substanzmissbrauch und Konsumproblemen (theoretisch) hauptsächlich infrage [4]:

- Erziehungsbeistand ( $\$ 30)$,

- Heimerziehung bzw. sonstige betreute Wohnformen (§34),

- intensive sozialpädagogische Einzelbetreuung (§35) sowie

- Eingliederungshilfe für seelisch behinderte Kinder und Jugendliche (§35a).

Diese vier Leistungsarten unterscheiden sich hinsichtlich des Unterstützungsbedarfs der Klienten und der Intensität in der Betreuung: vom „Erziehungsbeistand“ für Jugendliche ohne manifestierten Drogengebrauch bis hin zur „Eingliederungshilfe“ für suchtkranke Personen [4]. Dabei wird in vielen Kommentaren die fachliche Position vertreten, nach der eine bestehende Suchtkrankheit als eine seelische Behinderung aufgefasst werden kann [5]. Danach hätten Kinder und Jugendliche mit diesem Krankheitsbild Anspruch auf Eingliederungshilfe ( 335 a KJHG). Gleichzeitig wird vor einem inflationären Gebrauch dieser Rechtsauffassung gewarnt und auf die Gefahr der Stigmatisierung von jugendlichen Konsumenten hingewiesen [4]. Die beschriebenen Leistungen gelten auch für junge Erwachsene; für Personen ab 22 Jahren aber nur in begründeten Einzelfällen und für einen begrenzten Zeitraum ( $\$ 41 \mathrm{KJHG}$ ).

Vor dem dargestellten Hintergrund sollte die folgende Analyse gelesen werden. Dabei geht es nicht darum, einen Vorschlag für fachliche Zuständigkeiten oder für ein Kooperationsmodell zwischen der Sucht- und der Jugendhilfe zu unterbreiten, sondern es werden die jugendliche Klientel beschrieben und die von ihnen in Anspruch genommenen Leistungen dargestellt. Auf dieser Basis wird der Versuch einer (theoretischen) Zuordnung von Leistungen des KJHG vorgenommen. Dadurch soll eine empirische Grundlage für vertiefende Diskussionen über Formen der Kooperation und Vernetzung bzw. fachlich sinnvolle Abgrenzungen von Jugend- und Suchthilfe geschaffen werden.

\section{Datengrundlage und methodische Hinweise}

Als empirische Grundlage für die Analyse wurden Daten aus Schleswig-Holstein ausgewertet. Dort findet seit einigen Jahren 
ein systematisches Monitoring der Klienten und Tätigkeiten der ambulanten Suchthilfe statt [6, 7]. Dieses basiert auf einem EDVgestützten Dokumentationsprogramm (HORIZONT), das von den ambulanten Einrichtungen flächendeckend eingesetzt wird. Es liegt deshalb eine umfangreiche Datengrundlage vor, die Schleswig-Holstein für eine beispielhafte Analyse geeignet erscheinen lässt. Es sei angemerkt, dass in den Kreisen und kreisfreien Städten des Landes Schleswig-Holstein eine unterschiedlich intensive Zusammenarbeit zwischen der Jugend- und der Suchthilfe besteht. Aus diesem Grund dürfte sich hier eine vielfältige Praxis widerspiegeln, die es auch in anderen Teilen Deutschlands gibt.

Die empirische Basis dieser Analyse stellen die erfassten Informationen von 54 Einrichtungen der ambulanten Suchthilfe Schleswig-Holsteins im Zeitraum 1997 bis 2002 dar. In dieser Phase haben sich innerhalb der zu betrachtenden Altersgruppen keine wesentlichen Strukturverschiebungen ereignet. Bei den Einrichtungen handelt es sich größtenteils um integrative Suchtberatungsstellen, ferner um einige niedrigschwellige Angebote für Drogenkonsumenten aus den größeren Städten. Spezialisierte Beratungseinrichtungen für Jugendliche befinden sich nicht darunter.

Insgesamt sind Informationen von 14842 Klienten mit 13565 Betreuungen in diese Analyse eingeflossen. ${ }^{1}$ Diese Anzahl verteilt sich zwar - erwartungsgemäß - ungleich über die Altersgruppen; in jeder der betrachteten Gruppen ist jedoch eine hinreichende Anzahl von Fällen ( $\mathrm{n}$ ) vorhanden, so dass statistisch valide Auswertungen möglich sind. Ein Teil der Auswertungen basiert auf den Angaben zu den Klienten (n-Klienten), der andere auf Angaben zu den Betreuungen (n-Betreuungen).

In den folgenden Auswertungen werden drei Altersgruppen miteinander verglichen:

- die Gruppe "Jugendliche“ (14 bis 17 Jahre),

- die Gruppe ,junge Erwachsene“ (18 bis 26 Jahre) und

- die Gruppe „ältere Erwachsene“ (27 Jahre und älter).

Die letztgenannte Gruppe dient als Vergleichsgruppe. Eine Gruppe „Kinder“ (Personen unter 14 Jahren) konnte aufgrund einer zu geringen Fallzahl nicht gebildet werden. Ihr Gesamtanteil an allen Klienten beträgt 0,3\%.

Die folgende Analyse ist die erste dieser Art in Deutschland. Hier zeigen sich die Auswertungs- und Erkenntnismöglichkeiten, die mit einer flächendeckenden und langjährig angelegten EDV-gestützten Dokumentation verbunden sind.

\section{Anteil Jugendliche und junge Erwachsene}

Welchen Anteil weisen nun die jungen Personen an der Gesamtzahl der Klienten auf? Bei $5 \%$ aller Klienten handelt es sich um 14- bis 17-Jährige, d.h. jede zwanzigste Person, die eine ambulante Suchthilfeeinrichtung in Schleswig-Holstein aufsucht, ist

\footnotetext{
1 Der Sachverhalt, dass es weniger Betreuungen als Klienten gibt, erklärt sich daraus, dass in den ersten Jahren (97-99) noch keine Betreuungen dokumentiert worden sind.
}

minderjährig (siehe Abb.1). Der Anteil der jungen Erwachsenen (18 bis 26 Jahre) ist mit 19\% weit höher. Beide Gruppen junger Klienten zusammengenommen kommen damit immerhin auf einen Anteil von beinahe $25 \%$. Aufgrund dessen hat die Betreuung von Klienten, die sich in der Altersspanne des KJHG befinden, eine nicht unwesentliche Bedeutung für die ambulante Suchthilfe. Erst recht, wenn in Betracht gezogen wird, dass es sich hierbei um eine Klientel handelt, bei der viele Personen noch nicht langjährig schwerstabhängig sind, sondern häufig ein Missbrauchsverhalten vorliegt, bei dem Frühinterventionen und Präventionsmaßnahmen erforderlich sind.

Die Geschlechterverteilung ist in allen drei Altersgruppen sehr ähnlich: Bei den Jugendlichen sind 70\% männlichen Geschlechts, bei $30 \%$ handelt es sich um junge Frauen (siehe Abb.1). In den anderen beiden Altersgruppen sind die Männer sogar noch etwas stärker vertreten (jeweils 75\% zu 25\%).

\section{Kontaktempfehlung}

Deutliche Unterschiede zwischen den Altersgruppen zeigen sich, wenn der Frage nachgegangen wird, über wen die Klienten in die Einrichtung kommen. Es wird dann deutlich, dass der Kontakt von jugendlichen Klienten häufig aufgrund einer Initiative der Familie, der Freunde oder der Partner zustande kommt (38\%). Auch die Schule oder der Ausbildungsbetrieb spielen in dieser Altersgruppe als „Kontaktbahner“ mit einem Anteil von 19\% eine wichtige Rolle, während die professionelle Hilfe (andere Suchthilfeeinrichtungen, ärztlich-medizinischer Bereich) diesbezüglich kaum eine Bedeutung besitzt (zusammen $4 \%$ ). Nur jeder zehnte Jugendliche sucht aus eigener Motivation („ohne Vermittlung“) ein Hilfeangebot auf. Diese Zahlen zusammengenommen lassen die Interpretation $\mathrm{zu}$, dass ein nicht unerheblicher Teil der Jugendlichen von den „Erziehungsverantwortlichen“ (Familie, Schule) zu einer Suchtberatungsstelle geschickt wird, um dort pädagogische und/oder beraterische Unterstützung in Fragen von Sucht und Drogen zu erhalten (Erziehungsbeistand, $\S 30 \mathrm{KJHG}$ ).

Bei den jungen Erwachsenen stellt sich das Muster der Kontaktempfehlung anders dar: Fast ein Fünftel dieser Klienten gelangt über die professionelle Hilfe an die Einrichtung (zusammen $18 \%$ ); ein ebenso großer Anteil kommt aus eigenem Antrieb. Die

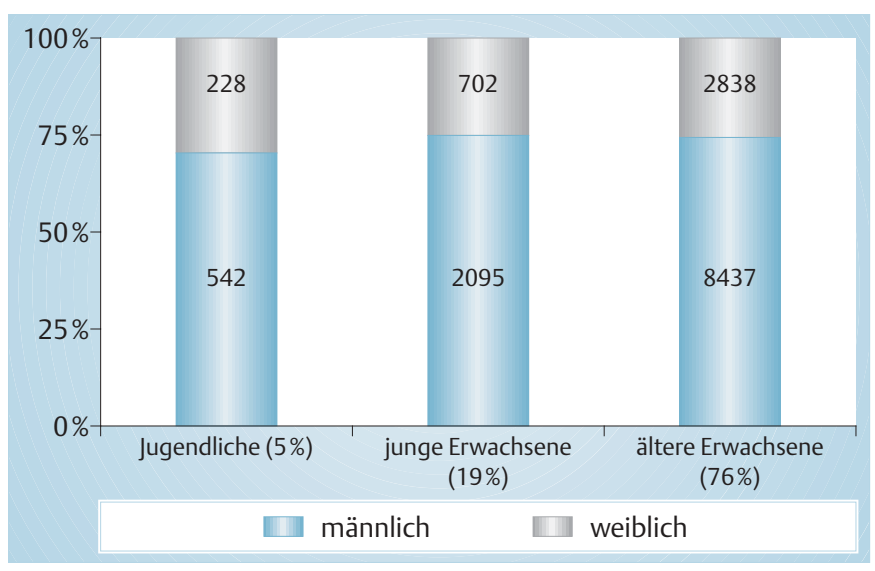

Abb. 1 Anzahl Jugendliche und junge Erwachsene nach Geschlecht. 
Tab. 1 Hauptdroge/-störung der Jugendlichen und jungen Erwachsenen

\begin{tabular}{|lccc|}
\hline & $\begin{array}{l}\text { Jugendliche } \\
\text { (14 bis 17 Jahre) }\end{array}$ & $\begin{array}{c}\text { junge Erwachsene } \\
\text { (18 bis 26 Jahre) }\end{array}$ & $\begin{array}{c}\text { (ältere) Erwachsene } \\
\text { (27 Jahre und älter) }\end{array}$ \\
\hline Alkohol & $16 \%$ & $21 \%$ & $67 \%$ \\
\hline Opiate & $12 \%$ & $43 \%$ & $26 \%$ \\
\hline Cannabis & $57 \%$ & $21 \%$ & $1 \%$ \\
\hline Ecstasy & $7 \%$ & $6 \%$ & $0,2 \%$ \\
\hline Glücksspiel & $0,4 \%$ & $3 \%$ & $3 \%$ \\
\hline Essstörungen & $8 \%$ & $7 \%$ & $2 \%$ \\
\hline n-Klienten & 770 & 2797 & 11275 \\
\hline
\end{tabular}

Bedeutung von Familie/Freunde und Betrieb/Schule für die Kontaktvermittlung lässt dagegen stark nach. In der Gruppe der (älteren) Erwachsenen setzt sich diese Tendenz verstärkt fort.

\section{Hauptdroge/-störung}

Hinsichtlich der Hauptdroge/-störung unterscheiden sich die Altersgruppen deutlich: Der Großteil der Jugendlichen hat wegen Cannabiskonsum (oder damit verbundener Probleme) die Einrichtung aufgesucht. Bei $57 \%$ aller 14- bis 17-Jährigen wird diese Substanz als Hauptdroge angegeben (siehe Tab.1). Die zweite „Jugenddroge“ Ecstasy kommt in dieser Altersgruppe auf einen Anteil von 7\%. Ungefähr jeder sechste Jugendliche hat ein Alkoholproblem (16\%); $12 \%$ sind Heroingebraucher. ${ }^{2}$

Die Gruppe der jungen Erwachsenen weist dagegen ein gänzlich anderes „Konsumprofil“ auf: Die meisten Klienten dieser Altersgruppe sind Opiatabhängige (43\%). Auch der Anteil der Personen mit der Hauptdroge Alkohol ist höher (21\%). Dagegen hat Cannabis nicht die zentrale Bedeutung wie bei den 14- bis 17-Jährigen (21\%). Die (relativen) Anteile der Ecstasy- und essgestörten Klienten sind dagegen in beiden jungen Altersgruppen beinahe gleich (7\% zu 6\% bzw. 8\% zu 7\%) (siehe Tab.1).

Werden die (älteren) Erwachsenen mit in die Betrachtung einbezogen, zeigt sich, dass je älter die Klienten sind, desto größer der Anteil der Alkoholabhängigen wird. Er beträgt bei den Personen, die über 26 Jahre alt sind, immerhin $67 \%$, d.h. zwei von drei Klienten dieser Altersgruppe kommen aufgrund einer Alkoholproblematik in eine Einrichtung.

Es zeigen sich, wenn die drei Gruppen miteinander verglichen werden, keine geschlechtsspezifischen Unterschiede bei der Hauptdroge/-störung: Beispielsweise sind in allen Altersgruppen die Cannabiskonsumenten überdurchschnittlich häufig Männer. Ihr Anteil liegt in den drei Gruppen zwischen $81 \%$ (ältere Erwachsene) und $88 \%$ (junge Erwachsene). Ferner bestätigt sich in allen Altersgruppen der bekannte Sachverhalt, dass es sich bei

2 Wegen einer bestehenden Nikotinabhängigkeit sucht kaum ein Jugendlicher oder ein junger Erwachsener eine ambulante Suchthilfeeinrichtung auf. Die Fallzahl ist hier kaum messbar; sie lag zum Beispiel für das Jahr 2002 unter 10 Personen (für beide Kategorien zusammengenommen). den essgestörten Personen überwiegend um Frauen handelt (Anteile zwischen $88 \%$ bis $94 \%$ ).

Wird eine Einschätzung versucht, inwieweit in diesem Bereich Voraussetzungen für Leistungen des KJHG erfüllt sein könnten, kann aufgrund des dargestellten hohen Anteils von Opiatabhängigen in der Gruppe der jungen Erwachsenen vermutet werden, dass zumindest (theoretisch) ein Teil dieser Personen die Voraussetzungen des $\S 35$ a KJHG (Eingliederungshilfen) erfüllen dürfte. $^{3}$ Erst recht, wenn berücksichtigt wird, dass immerhin $46 \%$ der jungen Erwachsenen das 22. Lebensjahr noch nicht vollendet haben und deswegen nicht unter die Ausnahmefallregelung des KJHG fallen würden (siehe oben). Das „Konsumprofil“ der Jugendlichen deutet dagegen eher daraufhin, dass hier präventive und pädagogisch unterstützende Hilfen des KJHG zum Tragen kommen könnten. ${ }^{4}$

\section{Soziale Situation}

Die soziale und biografische Situation stellt sich bei jeder der drei Altersgruppen unterschiedlich dar: Die Jugendlichen wohnen zu fast drei Viertel (noch) zu Hause (73\%) und werden meistens von den Angehörigen finanziert (77\%) (siehe Tab. 2). Fast zwei Drittel der 14- bis 17-Jährigen haben (noch) keinen festen Partner; sie bezeichnen sich als „allein stehend“ (65\%). Von denjenigen 14bis 17-Jährigen, die nicht mehr zur Schule gehen, hat mehr als jeder Vierte mindestens mit der mittleren Reife abgeschlossen.

Im Unterschied zu den Jugendlichen leben - erwartungsgemäß die jungen Erwachsenen schon viel häufiger in der eigenen Wohnung (42\%) und gehen einer Erwerbstätigkeit nach (37\%). Aber auch $43 \%$ dieses Personenkreises finanzieren ihren Lebensunterhalt durch Transferleistungen wie Arbeitslosengeld oder Sozialhilfe. Diese Quote entspricht der der (älteren) Erwachsenen. Erst jeder Fünfte der 18- bis 26-Jährigen verfügt über eine abgeschlossene Berufsausbildung. Bei den (älteren) Erwachsenen haben immerhin $60 \%$ eine Lehre oder Ausbildung abgeschlossen.

Es bestehen zwischen den Altersgruppen einige geschlechtsund substanzspezifische Unterschiede: Bei den jungen Erwachsenen verfügen $52 \%$ der Frauen über eine eigene Wohnung, bei den Männern sind dies nur 38\%. Dagegen wohnen diese noch häufiger bei den Eltern als ihre Mitklientinnen (38\% zu 29\%). Auch sind die männlichen 18- bis 26-Jährigen eher alleinstehend als die Frauen (56\% zu 38\%). Diese gravierenden Abweichungen existieren, obwohl beide Geschlechter im Durchschnitt exakt gleich alt sind (22,4 Jahre). Andererseits ist aber aus soziologi-

\footnotetext{
3 Es sei in diesem Zusammenhang darauf hingewiesen, dass es in der Praxis häufiger vorkommen dürfte, dass für volljährige Klienten Eingliederungshilfen (z.B. Betreutes Wohnen) nach Bundessozialhilfegesetz (BSHG §39/40) bei den zuständigen Kostenträgern beantragt werden.

4 Natürlich könnte auch bei dieser Klienten-Gruppe in Einzelfällen der $\S 35$ a KJHG zum Tragen kommen, da dieser schon gilt, wenn Kinder und Jugendliche von einer seelischen Behinderung bedroht sind (=Suchtgefährdung). Um hierzu eine genauere quantitative Abschätzung vornehmen zu können, müssten jedoch zusätzliche Informationen über die psychische Situation und die konkreten Konsummuster der Klienten vorliegen.
} 


\begin{tabular}{|c|c|c|c|}
\hline & $\begin{array}{l}\text { Jugendliche } \\
\text { (14 bis 17 Jahre) }\end{array}$ & $\begin{array}{l}\text { junge Erwachsene } \\
\text { (18 bis 26 Jahre) }\end{array}$ & $\begin{array}{l}\text { (ältere) Erwachsene } \\
\text { (27 und älter) }\end{array}$ \\
\hline \multicolumn{4}{|l|}{ Wohnsituation } \\
\hline eigene Wohnung & $5 \%$ & $42 \%$ & $82 \%$ \\
\hline bei den Eltern & $73 \%$ & $35 \%$ & $5 \%$ \\
\hline instabile Situation & $2 \%$ & $7 \%$ & $5 \%$ \\
\hline \multicolumn{4}{|l|}{ Partnerbeziehung } \\
\hline allein stehend & $65 \%$ & $50 \%$ & $38 \%$ \\
\hline feste Beziehung & $12 \%$ & $34 \%$ & $54 \%$ \\
\hline \multicolumn{4}{|l|}{ Schul- und Berufsbildung } \\
\hline mittlere Reife und höher & $28 \%$ & $30 \%$ & $34 \%$ \\
\hline Ausbildung abgeschlossen & 1 & $21 \%$ & $60 \%$ \\
\hline \multicolumn{4}{|l|}{ Haupteinkommensquelle } \\
\hline Erwerbstätigkeit & $16 \%$ & $37 \%$ & $43 \%$ \\
\hline Arbeitslosengeld/Sozialhilfe & $6 \%$ & $43 \%$ & $42 \%$ \\
\hline Angehörige & $77 \%$ & $18 \%$ & $7 \%$ \\
\hline n-Klienten & 475 & 1693 & 7916 \\
\hline
\end{tabular}

Tab. 2 Soziale Situation der Jugendlichen und jungen Erwachsenen ${ }^{1}$

${ }^{1}$ Die fehlenden Prozentwerte in der Tabelle betragen für die drei Altersgruppen (1: Jugendliche, 2: junge Erwachsene, 3: ältere Erwachsene):

a) Wohnsituation „andere“ $-1: 20 \%, 2: 17 \%, 3: 8 \%$

b) Partnerbeziehung „zeitweilige Beziehung“ - 1:23\%2: 16\%, 3: $8 \%$

c) Haupteinkommensquelle „anderes“ - 1:1\%, 2: 2\%, 3: $8 \%$

schen Untersuchungen bekannt, dass jüngere Frauen eher eine feste Beziehung (mit älteren Partnern) eingehen als jüngere Männer. Bei den Jugendlichen gibt es diese Unterschiede in dieser Form nicht. Auch die größte substanzspezifische Differenz findet sich in der Gruppe der jungen Erwachsenen. Hier sind in prozentualer Hinsicht doppelt so viele alkoholabhängige Personen erwerbstätig wie bei den Heroinabhängigen ( $46 \%$ zu $23 \%$ ). Umgekehrt finanziert sich die zweite Gruppe viel häufiger durch Arbeitslosengeld oder Sozialhilfe (62\% zu 38\%).

Insgesamt betrachtet kann festgehalten werden, dass jede Altersgruppe eine spezifische soziale und biografische Konstellation aufweist. Während die meisten Jugendlichen in sozialer Hinsicht mehr oder weniger integriert sein dürften, stellt sich die soziale Situation für einen erheblichen Teil der jungen Erwachsenen in einigen Punkten ähnlich negativ dar - z. B. bei der Erwerbssituation - wie bei den (älteren) Erwachsenen. Die sozial-biografischen Daten bestätigen also, dass zumindest ein Teil der 18- bis 26-Jährigen intensivere (psycho-)soziale Hilfen benötigt. Gleichzeitig könnte die Interpretation dieses Sachverhalts auch so ausfallen, dass Frühinterventionen eine sinnvolle Aufgabe darstellen, um spätere Verfestigungen zu vermeiden.

\section{In Anspruch genommene Leistungen}

Im Folgenden soll das Inanspruchnahmeverhalten der Jugendlichen und jungen Erwachsenen analysiert werden. Zeigen sich auch hier - wie schon bei der biografischen und sozialen Situation - Besonderheiten in den Altersgruppen und wie sind diese vor dem Hintergrund des KJHG einzuordnen?

Zunächst einmal wird deutlich, dass Jugendliche vor allem beraten werden, wenn sie eine Suchthilfeeinrichtung aufsuchen. Das trifft auf neun von zehn Jugendlichen zu (93\%). Therapeutische Leistungen, wie beispielsweise die Substitutionsbehandlung oder die ambulante Rehabilitation, spielen kaum eine Rolle (7\%).
Vor dem Hintergrund des Konsumprofils und der sozialen Situation dieser Altersgruppe war dies auch nicht anders zu erwarten. Auffällig ist aber, dass nur bei ganz wenigen der 14- bis 17-Jährigen Vermittlungsleistungen erbracht werden (4\%). ${ }^{5}$

Bei den jungen Erwachsensen nimmt dagegen schon beinahe jeder vierte Klient therapeutische Leistungen in Anspruch (22\%). Dies hängt mit dem hohen Anteil von Heroinabhängigen in dieser Altersgruppe zusammen (siehe oben). Auch der Anteil von Klienten mit Vermittlungsleistungen steigt deutlich an (21\%). Das Profil der in Anspruch genommenen Leistungstypen verändert sich von den jungen zu den älteren Erwachsenen nur wenig.

Wird das Inanspruchnahmeverhalten weiter analysiert, zeigt sich, dass die Jugendlichen am wenigsten Leistungen nachfragen: Sie kommen (im Durchschnitt) fünfmal in eine Einrichtung und lassen sich dabei insgesamt 3,4 Stunden betreuen und beraten (siehe Tab. 3). Bei den jungen Erwachsenen betragen die entsprechenden Werte schon dreizehn Kontakte mit einem durchschnittlichen Zeitumfang von 8,6 Stunden. Bei den (älteren) Erwachsenen nimmt die Intensität der Betreuung weiter und sehr deutlich zu.

Auch die Betreuungslänge - also der Wert dafür, wie lange eine Betreuungssequenz dauert - nimmt von Altersgruppe zu Altersgruppe zu: Die jungen Erwachsenen sind im Mittel fast doppelt so lange in Betreuung wie die Jugendlichen (163 zu 85 Tage). Bei den (älteren) Erwachsenen nimmt die durchschnittliche Betreu-

\footnotetext{
5 Der angegebene Wert sagt nichts darüber aus, ob die Vermittlung tatsächlich stattgefunden hat oder nicht. Es geht hier um den Leistungstyp. Außerdem ist anzumerken, dass die abweichenden Prozentangaben im Vergleich zu den noch folgenden Auswertungen zu den Vermittlungen (siehe weiter unten) aufgrund unterschiedlicher Fallzahlen und Dokumentationsbereiche (Leistungskatalog, Behandlungsmodul) zustande kommen. Die Grundaussagen sind aber bei beiden Auswertungen identisch und empirisch valide.
} 


\begin{tabular}{|c|c|c|c|}
\hline & $\begin{array}{l}\text { Jugendliche } \\
\text { (14 bis17 Jahre) }\end{array}$ & $\begin{array}{l}\text { junge Erwachsene } \\
\text { (18 bis } 26 \text { Jahre) }\end{array}$ & $\begin{array}{l}\text { (ältere) Erwachsene } \\
\text { (27 und älter) }\end{array}$ \\
\hline Anzahl & 5 & 13 & 20 \\
\hline Dauer insgesamt & 3,4 Std. & 8,6 Std. & 17,2 Std. \\
\hline Dauer pro Leistung & $41 \mathrm{Min}$. & 40 Min. & 52 Min. \\
\hline n-Klienten & 661 & 2552 & 10711 \\
\hline
\end{tabular}

Tab. 3 Anzahl und Dauer der von den Jugendlichen und jungen Erwachsenen in Anspruch genommenen Leistungen (Mittelwerte) ungslänge noch einmal um etwa ein Drittel zu (226 Tage). Insgesamt gesehen sind also die Jugendlichen deutlich kürzer, mit weniger Kontakten und geringerem Zeitaufwand in einer Betreuung als die jungen Erwachsenen.

Werden die Anzahl und die Dauer der erbrachten Leistungen sowie die Länge der Betreuung als Indikatoren für Hilfebedarfe angesehen, können die Jugendlichen eher als eine Gruppe klassifiziert werden, die eine ergänzende Unterstützung (im Sinne des Erziehungsbeistands) benötigt, während für die Gruppe der jungen Erwachsenen eine strukturierte, intensive und problemorientierte Betreuung (im Sinne der Intensiven Sozialpädagogischen Einzelbetreuung oder der Eingliederungshilfe) erforderlich ist.

Differenziert nach dem Hauptdrogentyp/(-störungstyp) ergeben sich in dieser Hinsicht weitere Erkenntnisse: Die geringste Betreuungszeit - von allen Altersgruppen und allen Hauptdrogentypen - haben die jugendlichen Cannabiskonsumenten, die die dominierende Gruppe bei den 14- bis 17-Jährigen darstellen. Sie kommen auf 1,9 Stunden Betreuung im Durchschnitt, d. h. sie suchen in der Regel ein- bis dreimal eine Suchthilfeeinrichtung auf. In dieser Altersgruppe verursachen die essgestörten Personen den größten zeitlichen Aufwand: 7,8 Stunden je Klient. Offensichtlich besteht hier die Notwendigkeit, schon mit sehr jungen Menschen strukturiert und zeitintensiv zu arbeiten.

Bei den jungen Erwachsenen ist - neben den Essgestörten - die Betreuung der Opiatabhängigen sehr zeitaufwändig. Sie fragen in zeitlicher Hinsicht fast doppelt so viele Leistungen nach wie ihre alkoholabhängigen Mitklienten (12,1 zu 6,4 Stunden). Bei den (älteren) Erwachsenen hat sich das Inanspruchnahmeverhalten dieser beiden Klientengruppen auf einem sehr hohen $\mathrm{Ni}$ veau wieder angeglichen (17,6 zu 17,5 Stunden).

Die Analyse der Betreuungslänge nach dem Hauptdrogentyp ergänzt die obigen Befunde. Die jugendlichen Cannabiskonsumenten bleiben vergleichsweise kurz: 81 Tage im Mittel, in denen sie beraten und betreut werden (dabei kommt es nur zu wenigen Kontakten, siehe oben). Gleichzeitig wird aber in dieser Betrachtungsweise deutlich, dass auch die anderen jugendlichen Konsumentengruppen nicht wesentlich länger in Betreuung bleiben, obwohl sie vom zeitlichen Umfang her mehr Leistungen empfangen. Möglicherweise hängt dies mit einem jugendspezifischen Beendigungsverhalten zusammen (siehe unten).

Bei den jungen Erwachsenen nimmt die Betreuungslänge insgesamt zu; hier zeigen sich darüber hinaus aber auch deutliche substanzspezifische Unterschiede: Die Betreuung dauert mit Abstand am längsten bei den Opiatabhängigen (durchschnittlich 202 Tage), bei den anderen Konsumentengruppen liegt dieser Wert zwischen 116 und 138 Tagen. Eine breite Streuung - bei ei- ner insgesamt weiter erhöhten Betreuungslänge - findet man bei den (älteren) Erwachsenen.

Insgesamt zeigen sich damit für jede der Konsumentengruppen sehr spezielle Inanspruchnahmemuster, wenn die Dauer und die Länge der Leistung/Betreuung zusammen betrachtet werden. Die bis zu diesem Punkt vorgenommene Analyse des Inanspruchnahmeverhaltens bestätigt die schon vorgenommene Einschätzung, dass bei einem Großteil der Jugendlichen - vorwiegend den Cannabiskonsumenten - eine von ihrem Charakter her präventiv und pädagogisch ausgerichtete Kurzberatung durchgeführt wird, während es vor allem bei den jungen Erwachsenen die Gruppe der Opiatabhängigen ist, bei der eine intensive, teilweise therapeutisch orientierte Betreuung stattfindet.

\section{Inhalte der Beratung}

In der ambulanten Suchthilfe in Schleswig-Holstein werden auch die Inhalte der Beratung und die dafür aufgewendete Zeit EDVgestützt erfasst. Diese können Indikatoren für spezifische Unterstützungsbedarfe sein. Bei der statistischen Auswertung der entsprechenden Informationen wird deutlich, dass die Jugendlichen vergleichsweise häufig zum Thema „Sucht“ beraten werden. Jede vierte Beratungsminute wird dieser Problematik gewidmet. Dies deutet darauf hin, dass die Beratung Jugendlicher häufiger die Funktion einer allgemeinen Drogen- und Suchtaufklärung besitzt. Konkrete Behandlungsfragen (z. B. angemessene Therapieform) spielen dagegen in dieser Altersgruppe kaum eine Rolle. Es fällt weiter auf, dass Beziehungsfragen - seien es die Probleme mit der Familie, den Freunden oder dem Partner - eine vergleichsweise große Bedeutung haben.

Das „Beratungsprofil“ der jungen Erwachsenen ähnelt dagegen stark dem der Gruppe der (älteren) Erwachsenen: Die relative Bedeutung der Themen „Sucht“ und „Beziehung“ nimmt ab, die von Behandlungsfragen zu.

Wesentliche geschlechtsspezifische Unterschiede zwischen den Altersgruppen gibt es bei den Beratungsinhalten nicht. Beispielsweise spielt in allen drei Gruppen das Thema „Beziehung“ bei den Frauen eine größere Rolle als bei den Männern.

Zusammenfassend betrachtet können die dargestellten Ergebnisse derart interpretiert werden, dass die Beratung Jugendlicher zumindest teilweise den Charakter einer allgemeinen erzieherischen Beratung hat, wenn diese an den Beratungsinhalten „Sucht“ und „Beziehung“ gemessen wird. In dieser Hinsicht bestehen zumindest inhaltliche Anknüpfungspunkte zu dem im KJHG definierten Aufgabenspektrum der Jugendhilfe. 
Betreuungsende und Konsumstatus

Die Ergebnisse zum Betreuungsende weisen einige Besonderheiten auf: Die Jugendlichen beenden von allen drei Altersgruppen am häufigsten planmäßig ihre Betreuung (44\%) (siehe Tab.4). Gleichzeitig brechen aber immerhin genauso viele der 14- bis 17-Jährigen - egal ob männlichen oder weiblichen Geschlechts - die Betreuung von sich aus ab. Überdurchschnittlich oft ist dies bei Ecstasy-Konsumenten (62\%) und Opiatabhängigen (51\%) der Fall. Bei der Gruppe der jungen Erwachsenen liegt die Abbruchquote sogar bei $50 \%$. Hier finden sich solch hohe Werte bei allen Hauptdrogentypen/(-störungstypen), mit Ausnahme der Essstörungen (40\%). Auffällig ist ferner, dass die Jugendlichen im Vergleich zu den anderen beiden Altersgruppen seltener weitervermittelt werden ( $8 \% \mathrm{zu} 14 \%$ ), was mit der höheren Quote planmäßiger Beendigungen zusammenhängen dürfte.

Wenn Klienten am Ende der Betreuung weitervermittelt werden, sind kaum Unterschiede zwischen den Altersgruppen bei der Frage erkennbar, wohin sie vermittelt werden: Ein erheblicher Anteil von ihnen wird in die Entzugsbehandlung oder in eine stationäre Entwöhnungstherapie weitergeleitet (38\% bis $46 \%$ ). In eine ambulante Beratungs- oder Therapieform wird jeweils etwa jede sechste (weitervermittelte) Person geschickt. Andere Betreuungsformen (wie betreutes Wohnen, Nachsorge etc.) besitzen in allen Altersgruppen nur eine nachgeordnete Bedeutung.

Die Konsumsituation zum Zeitpunkt der Beendigung der Betreuung stellt sich ambivalent dar: Die Jugendlichen weisen zwar die geringste Abstinenzrate von $15 \%$ auf. Gleichzeitig verbessert sich bei jeder dritten Person dieser Altersgruppe die Konsumsituation (33\%). Bei jedem zweiten Jugendlichen bleibt diese unverändert. Diese Zahlen dürften sich daraus erklären, dass ein Großteil der 14- und 17-Jährigen nicht abhängig ist und aufgrund dessen die Abstinenz nicht bei allen Klienten das primäre Ziel der Betreuung darstellt.

Die „Erfolgsquote“ der Betreuung - die Kategorien „abstinent“ und „gebessert“ zusammen gerechnet - steigt mit zunehmendem Alter an: in der Gruppe der jungen Erwachsenen beträgt sie $50 \%$, bei den älteren Erwachsenen $62 \%$. Bei der ersten Altersgruppe gibt es hierbei keinen Unterschied zwischen den Opiatund Alkoholabhängigen. Bei der zweiten existiert dagegen eine gravierende Abweichung: Während $43 \%$ der alkoholabhängigen Personen am Ende der Betreuung als „abstinent“ eingestuft werden, beträgt dieser Anteil bei den Heroinabhängigen „nur“ $20 \%$. Geschlechtsspezifische Differenzen zwischen den drei Altersgruppen bestehen nicht.
Werden der Abschluss der Betreuung und der dort bestehende Konsumstatus zusammen betrachtet, zeigt sich, dass sich bei einem erheblichen Anteil der Jugendlichen mit einer planmäßigen Beendigung der Konsum nicht verändert hat (40\%). Dieser Anteil liegt in den anderen beiden Altersgruppen wesentlich niedriger: 29\% (junge Erwachsene) und 15\% (ältere Erwachsene).

Dieser Sachverhalt sollte nicht als eine erfolglose Betreuung Jugendlicher missinterpretiert werden. Vielmehr könnte hierin ein Indiz dafür gesehen werden, dass bei der Beratung dieser Altersgruppe nicht immer die Abstinenz (oder zumindest die Konsumreduktion) als Zielsetzung im Vordergrund steht, sondern die Suchthilfe hier vorbeugende, pädagogische Aufgaben im Sinne einer Verhinderung des Abgleitens in eine Abhängigkeit wahrnimmt.

\section{Schlussfolgerungen}

Die zentralen Ergebnisse der vorangegangenen empirischen Auswertung können wie folgt zusammengefasst werden: Bei 5\% der Klienten der ambulanten Suchthilfe handelt es sich um Jugendliche (14 bis 17 Jahre) und bei 19\% um junge Erwachsene (18 bis 26 Jahre). Die „Konsumprofile“ der Jugendlichen und jungen Erwachsenen weichen stark voneinander ab. Bei der erstgenannten Gruppe dominieren die Cannabisgebraucher, in der zweiten gibt es viele Heroinabhängige. Entsprechend stellt sich die soziale Situation dar: Die meisten Jugendlichen sind (noch) sozial integriert; bei den 18- bis 26-Jährigen befindet sich dagegen ein erheblicher Teil in einer schlechten sozialen Lage - u.a. verfügen sie über keine abgeschlossene Berufsausbildung und sind arbeitslos. Ebenfalls ist das Inanspruchnahmeverhalten beider Gruppen verschieden: Die Jugendlichen sind deutlich kürzer, mit weniger Kontakten und geringerem Zeitaufwand in einer Betreuung als die jungen Erwachsenen.

Vor diesem Hintergrund würde deshalb eine (theoretische) Zuordnung der Klientel und der empfangenen Hilfen zum Leistungskatalog des Kinder- und Jugendhilfegesetzes (KJHG) wie folgt ausfallen: Da es sich bei einem Großteil der Jugendlichen um Personen handelt, die (höchstens) suchtgefährdet, aber nicht abhängig sind, nehmen die Suchtberatungsstellen hier präventive und frühintervenierende Aufgaben wahr. Hierbei dürfte es häufig auch um Hilfestellungen zur Förderung der Persönlichkeitsentwicklung und zur Stärkung einer eigenverantwortlichen Lebensführung gehen. Beides sind Zielsetzungen, die auch im KJHG formuliert werden. Diese Leistungen wären am ehesten als unterstützende Leistungen im Sinne des Erziehungsbeistands

\begin{tabular}{lccc}
\hline & $\begin{array}{l}\text { Jugendliche } \\
\text { (14 bis 17 Jahre) }\end{array}$ & $\begin{array}{l}\text { junge Erwachsene } \\
\text { (18 bis 26 Jahre) }\end{array}$ & (ältere) Erwachsene (27 Jahre und älter) \\
\hline planmäßig & $44 \%$ & $30 \%$ & $37 \%$ \\
\hline Abbruch durch Klient/in & $44 \%$ & $50 \%$ & $42 \%$ \\
\hline Weitervermittlung & $8 \%$ & $14 \%$ & $14 \%$ \\
\hline anderes & $4 \%$ & $6 \%$ & $7 \%$ \\
\hline n-Betreuungen & 558 & 2209 & 9519 \\
\hline
\end{tabular}

Tab. 4 Betreuungsende der Jugendlichen und jungen Erwachsenen 
(§30) zu klassifizieren. Anders verhält es sich mit der Gruppe der jungen Erwachsenen: Hierunter befindet sich zumindest ein Teil von Heroinabhängigen (in kleinerem Umfang auch alkoholabhängige Personen), die als schwer abhängig, deswegen als suchtkranke Personen einzustufen sind. Dieser Personenkreis könnte unter §35a des KJHG - Eingliederungshilfen für seelisch behinderte Menschen - fallen. Wenn andere Optionen wie eine Behandlung auf der Grundlage des Bundessozialhilfegesetzes (BSHG §39/40) nicht infrage kommen, wäre dies möglicherweise der rechtliche und finanzielle Rahmen für eine Hilfeleistung. Dafür spricht auch die aufgezeigte Betreuungsintensität bei dieser Klientel. In diesem Zusammenhang wäre auch zu diskutieren, inwieweit eine verstärkte Frühintervention das Missverhältnis „wenige frühere Problemfälle zu vielen späteren Problemfällen“ mildern könnte.

Eine mögliche Begrenztheit der Aussagekraft der vorangegangenen Analyse könnte darin liegen, dass die präsentierten Ergebnisse nicht durch ein spezielles Forschungsprojekt, sondern im Rahmen eines EDV-gestützten Suchthilfe-Monitoring gewonnen worden sind. Die Validität der Daten wurde jedoch durch umfangreiche Plausibilitätstests sorgfältig geprüft. Ferner wurden bei der Erfassung der Daten keine anspruchsvolleren Beurteilungsinstrumente eingesetzt, die besondere Kenntnisse erfordern, so dass insgesamt von einer guten Datenqualität auszugehen ist. Davon abgesehen steht es natürlich außer Frage, dass speziellere Erhebungen und Untersuchungen zu diesem Themenkomplex weiterführende Ergebnisse liefern können. Aus diesem Grund soll mit diesem Beitrag ausschließlich eine empirische Grundlage für weitere Diskussionen über die Zusammenarbeit und Aufgabenverteilung von Suchthilfe und Jugendhilfe sowie die Weiterentwicklung spezifischer Angebote geschaffen werden. Mit dieser Analyse soll kein Vorschlag präferiert werden, wie zukünftig bestimmte Leistungen der Suchthilfe zu finanzieren sind. Dies setzt vielmehr einen fachlichen Konsens zwischen der Sucht- und der Jugendhilfe sowie den zuständigen Kostenträgern inklusive der Politik voraus.

\section{Literatur}

${ }^{1}$ Internationale Gesellschaft für erzieherische Hilfen (IGFH) (Hrsg). Dialog und Kooperation von Jugendhilfe und Drogenhilfe. Dokumentation eines ExpertInnengesprächs. Frankfurt a. M: Eigenverlag, 2001

${ }^{2}$ Eckert D, Bathen D (Hrsg). Jugendhilfe und akzeptierende Drogenarbeit. Freiburg i. B.: Lambertus, 1995

${ }^{3}$ Eckstein G. Kooperation von Drogenhilfe und Jugendhilfe aus der Sicht der Drogenhilfe. In: Internationale Gesellschaft für erzieherische Hilfen (IGFH) (Hrsg). Dialog und Kooperation von Jugendhilfe und Drogenhilfe. Dokumentation eines ExpertInnengesprächs. Frankfurt a. M.: Eigenverlag, 2001

${ }^{4}$ Schöne S. Drogen und Jugendhilfe. In: Böllinger L, Stöver H (Hrsg). Drogenpraxis-Drogenrecht-Drogenpolitik. Handbuch für Drogenbenutzer, Eltern, Drogenberater, Ärzte und Juristen. Frankfurt a. M.: Fachhochschulverlag, 2002

${ }^{5}$ Weber HJ. Kinder- und Jugendhilfegesetz (KJHG) und Drogenarbeit. Neue Möglichkeiten des Jugendamts aus Sicht einer Jugendhilfeeinrichtung. In: Eckert D, Bathen D (Hrsg). Jugendhilfe und akzeptierende Drogenarbeit. Freiburg i. B.: Lambertus, 1995

${ }^{6}$ Raschke P, Kalke J, Martens MS. Analysen der Inanspruchnahme. Moderne Dokumentation in der ambulanten Suchtkrankenhilfe (Band II). Kiel: Eigenverlag, 2002

${ }^{7}$ Kalke J, Martens MS, Schütze C et al. Die Lebenssituation der Klientinnen und Klienten in Stadt und Land. Moderne Dokumentation in der ambulanten Suchtkrankenhilfe (Band III). Kiel: Eigenverlag, 2003 